\title{
Taking the CCDs to the ultimate performance for low threshold experiments
}

\author{
Miguel Sofo Haro*t \\ Centro Atomico Bariloche - Instituto Balseiro, CNEA/CONICET, Argentina \\ E-mail: miguelsofoharo@cab.cnea.gob.ar
}

\section{Guillermo Fernandez Moroni}

Depto. de Ing. Electrica y de Computadores, Universidad Nacional del Sur, Bahia Blanca, Argentina

\section{Javier Tiffenberg}

Fermi National Accelerator Laboratory, Batavia, IL, United States

\section{Gustavo Cancelo}

Fermi National Accelerator Laboratory, Batavia, IL, United States

\section{Juan Estrada}

Fermi National Accelerator Laboratory, Batavia, IL, United States

\section{Xavier Bertou}

Centro Atomico Bariloche - Instituto Balseiro, CNEA/CONICET, Argentina

\section{Eduardo Paolini}

Depto. de Ing. Electrica y de Computadores, Universidad Nacional del Sur, Bahia Blanca, Argentina

\begin{abstract}
Scientific grade CCDs show atractive capabilities for the detection of particles with small energy deposition in matter. Their very low threshold of approximately $40 \mathrm{eV}$ and their good spatial reconstruction of the event are key properties for currently running experiments: CONNIE and DAMIC. Both experiments can benefit from any increase of the detection efficiency of nuclear recoils at low energy. In this work we present two different approaches to increase this efficiency by increasing the SNR of events. The first one is based on the reduction of the readout noise of the device, which is the main contribution of uncertainty to the signal measurement. New studies on the electronic noise from the integrated output amplifier and the readout electronics will be presented together with result of a new configuration showing a lower limit on the readout noise which can be implemented on the current setup of the CCD based experiments. A second approach to increase the SNR of events at low energy that will be presented is the studies of the spatial conformation of nuclear recoil events at different depth in the active volume by studies of new effects that differ from expected models based on not interacting diffusion model of electrons in the semiconductor.
\end{abstract}

38th International Conference on High Energy Physics

3-10 August 2016

Chicago, USA 


\section{Introduction}

CCDs were invented in 1969, and since there, their primary application field have been science, mostly astronomy and astrophysics. The development of fully depleted back side illuminated thick CCDs up to $675 \mu \mathrm{m}$, has extended its use to the detection of photons in the infrared region. This kind of CCD can be readout with a noise of $\approx 2 e-$, and can be up to $16 \mathrm{Mpix}$ making their mass $\approx 5 \operatorname{gr}[1]$. The last two characteristics, have motivated the application of the CCD bulk silicon as target material for low energy threshold particle experiments. Two pioneer experiments using this technology are CONNIE $[2,3]$ and DAMIC $[4,5,6]$. CONNIE stand for coherent neutrinonucleus interaction experiments, and aims at making the first detection of coherent scattering of antineutrinos with the nucleus of the silicon atoms of the CCDs. DAMIC stand for Dark Matter in CCDs, is a dark matter direct search experiment running at SNOLAB focused on the search for Weakly Interactive Massive Particles (WIMPs). The detection principle of both experiments is similar and it is as follows: the neutrino or WIMP will collide with a Si nucleus, and the nucleus recoil will produce e-h pairs. The holes are drifted by the electric field in the CCD bulk and collected in the potential well of the pixels. The expected recoil energy is below $1 \mathrm{keV}$ [2], equivalent to a range of $<2 \mu \mathrm{m}$, producing all the e-h pairs in a small volume compared to the pixel size $(15 \times 15 \mu \mathrm{m})$. We, therefore, call these events: point-like event. The events charge will be less than $100 e^{-}$as only a fraction of the recoil energy is converted in e-h pairs [4].

The CCDs used in DAMIC/CONNIE and in this work are developed by Lawrence Berkeley National Lab. They are $675 \mu \mathrm{m}$ thick, and made with high resistivity silicon $(>17 \mathrm{~K} \Omega \mathrm{cm})$, which leads to fully depleted operation at reasonably low values of substrate voltage $[7,8]$.

In section 2 we will show experimental evidence that the size of the point-like event depends on the amount of generated charge due to charge repulsion effects. A proper understanding of point-like events is very important because measuring its size in the images is possible to estimate its depth in the bulk and apply depth cuts to improve the signal-to-background ratio[6].

In section 3 we will show that the DAMIC/CONNIE CCDs are operated in order to limit the production of spurious charge. The only source of noise therefore from the readout electronics. To increase the detector efficiency, the readout noise must be reduced[4, 5, 6]. We will give a description of the electronic readout chain, and a new front-end electronics with a lower readout noise will be presented.

\section{Point-like Events}

To characterize point-like events we exposed the $\mathrm{CCD}$ to $\mathrm{X}$-rays, as they generate charge in a small volume (less than $1 \mu m[9,10]$ ), just like nuclear recoils. Figure 1 shows the image produced by three different X-rays energies that interacts near the back of the CCD. While the charge is generated in a volume smaller than the pixel size, the readout charge is spread over several pixels. This effect is attributed to the diffusion of the charge while it is drifted to the pixel collection well[11,2]. If only diffusion is considered, the size the events only depends on how far they occurs from the collection well $[6,11]$, and is not related with the charge generated by the event. The

\footnotetext{
${ }^{*}$ Speaker.
} 


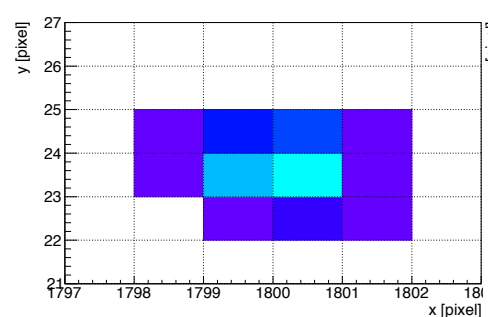

(a)

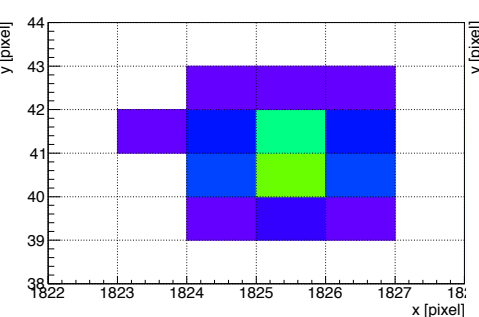

(b)

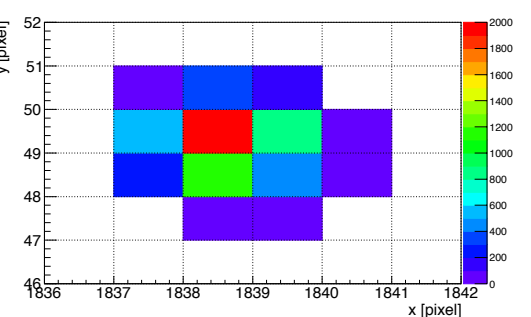

(c)

Figure 1: Image produced by three $\mathrm{X}$-rays energies (a) $8.04 \mathrm{KeV}$ (b) $13.39 \mathrm{KeV}$ (b) $22.16 \mathrm{KeV}$, from Copper $(\mathrm{Cu})$, Lead $(\mathrm{Pb})$ and Silver $(\mathrm{Ag})$ fluorescence respectively. Using the conversion factor of $3.77 \mathrm{eV} / \mathrm{e}^{-}$[6] they generates on average $2134 e^{-}, 3553 e^{-}$and $5878 e^{-}$respectively. All the pixels that have more that three times the electronic readout noise $\left(3 \sigma_{R} \approx 6 e^{-}\right)$are considered part of the event and form a cluster. The color scale is in $e^{-}$

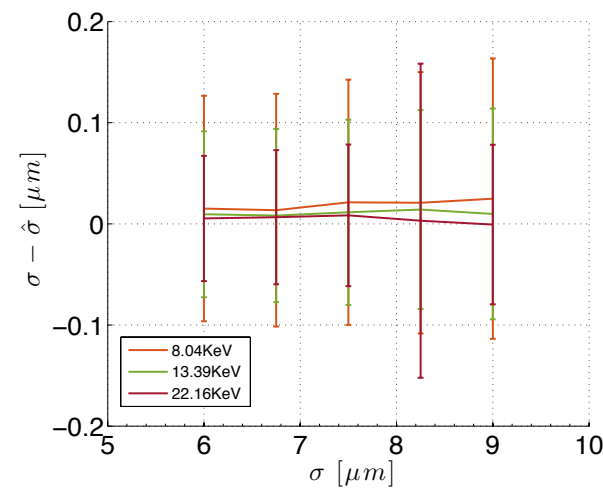

(a)

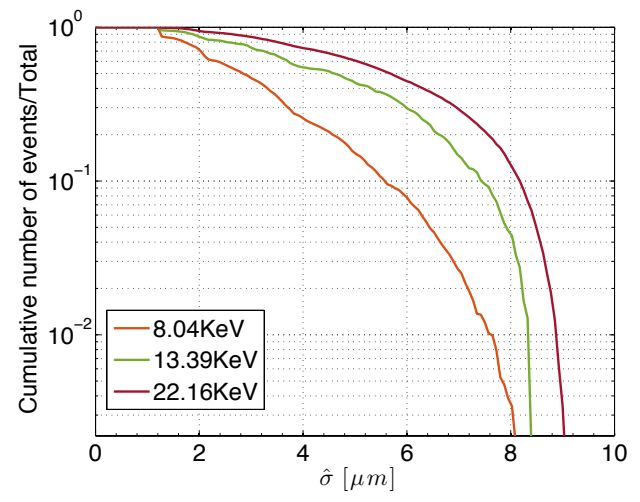

(b)

Figure 2: (a) Errors of the MLE of $\sigma$ (b) Cumulative distribution of $\hat{\sigma}$, the maximum $\hat{\sigma}$ for $8.04 \mathrm{KeV}$, $13.39 \mathrm{KeV}$ and $22.16 \mathrm{KeV}$ are $8 \mu \mathrm{m}, 8.33 \mu \mathrm{m}$ and $9 \mu \mathrm{m}$

spread of the events is measured as the $\sigma$ parameter of a two-dimensional Gaussian distribution, as is expected from the diffusion process [11].

A maximum likelihood estimator (MLE) of $\sigma$ had been implemented, it includes the "pixelation" effect and readout noise. Events with the energy of Copper $(\mathrm{Cu})$, Lead $(\mathrm{Pb})$ and Silver (Ag) $K_{\alpha}$ X-rays fluorescence peak have been simulated with different $\sigma$ to study the errors of the estimator after using the event clustering method, the results in figure2(a) shows that the MLE do not introduce a bias in the reconstructed $\sigma$ and the error is less than $0.2 \mu \mathrm{m}$

The CCD were exposed to collimated X-rays, with an angle $<3^{\circ}$, from the fluorescence of $\mathrm{Cu}, \mathrm{Pb}$ and $\mathrm{Ag}$. The plot in figure 2(b) is the cumulative distribution of the $\sigma$ parameter for the three different X-rays energies. For each X-ray the maximum spread $\left(\hat{\sigma}_{M A X}\right)$ is different, showing a dependence of $\sigma$ with the amount of charge generated by the X-ray. This effect has been observed and characterized for silicon drift detectors (SDD) [12, 13] and pnCCD [14]. It is due to charge repulsion and the size of the primary charge cloud formed by the X-rays. 


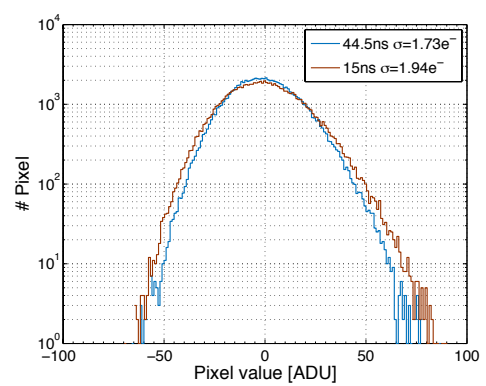

(a)

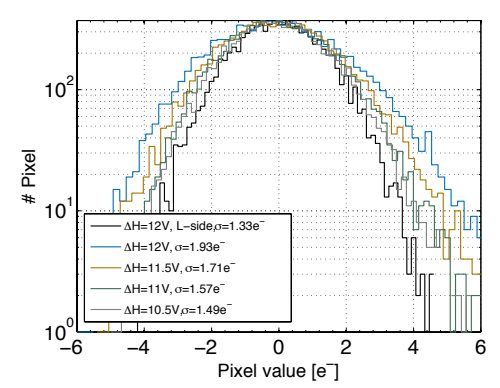

(b)

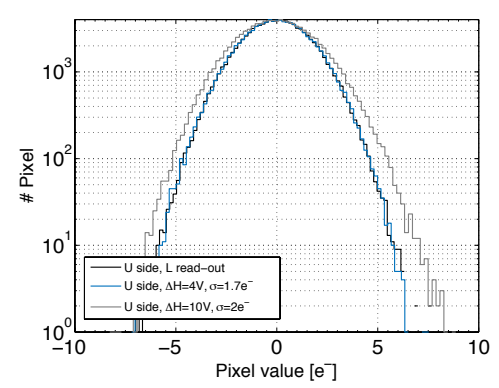

(c)

Figure 3: (a) Overscan pixel distribution of a $250 \mu \mathrm{m}$ CCD for two rise/fall times of the horizontal clock signals, the slowest one generate less spurious charge. (b) Overscan pixel distribution of the U-side amplifier of a $250 \mu \mathrm{m}$ CCD for different horizontal clock swing $(\Delta H)$, the minimum noise in the U-side is achieved when the CCD pixel array is readout through the L-side amplifier, hence the U-side have only electronic readout noise (c) Overscan pixel distribution of a $675 \mu \mathrm{m}$ CCD for two set of horizontal clock voltages, for the optimal set of voltages the noise of the U-side amplifier is the same if the CCD is readout by the L-side or U-side, this indicates that there is no generation of spurious charge.

\section{Noise in CCDs}

In particle detection applications of CCD's there are three main sources of noise. One of them is the noise added to the signal during the CCD exposure, $\sigma_{D}^{2}$, such as dark current and light leakage. A second source of noise, $\sigma_{S}^{2}$, is the spurious charge that is generated during the clocking of the CCD phases $[15,16,17]$. The last source of noise, $\sigma_{R}^{2}$, is added by the read-out electronics. These three sources are uncorrelated between them, and, as is shown in equation 3.1, they are added in quadrature. Each source can be measured using the method described in [18].

$$
\sigma_{T}^{2}=\sigma_{D}^{2}+\sigma_{S}^{2}+\sigma_{R}^{2}
$$

In CONNIE and DAMIC experiments the CCDs are cooled down to $\approx 120 \mathrm{~K}$, reducing the dark current level below $0.001 e^{-}$pix $^{-1}$ day $^{-1}$ [6]. Exposures up to several days can be taken without introducing significant noise from dark current.

Applying the technique described in [18], generation of spurious charge was observed in two types of fully depleted CCDs: the $250 \mu \mathrm{m}$ version used during the engineering run of CONNIE [3], and in the $675 \mu \mathrm{m}$ version currently used in CONNIE and DAMIC experiments [6]. A explanation on how spurious charge is generated can be found in [15]. In CONNIE/DAMIC CCD has been observed that the amount of spurious charge depends on the rise-fall time of the clock (figure 3(a)), and the voltage swing of the horizontal clock signal (figure 3(b)). An optimization of clock voltages for the $675 \mu \mathrm{m}$ CCDs was done to eliminate the generation of spurious charge. As figure 3 shows, DAMIC/CONNIE CCDs are only affected by electronic readout noise.

\subsection{Electronic Read-Out Noise}

Figure 4 shows the block diagram of a general readout chain of a CCD. If an amount of charge $\mathrm{Q}$ is placed in the sense node for its measurement, the voltage $V_{Q}$ at the gate of M1 will be $Q / C_{T}$, 


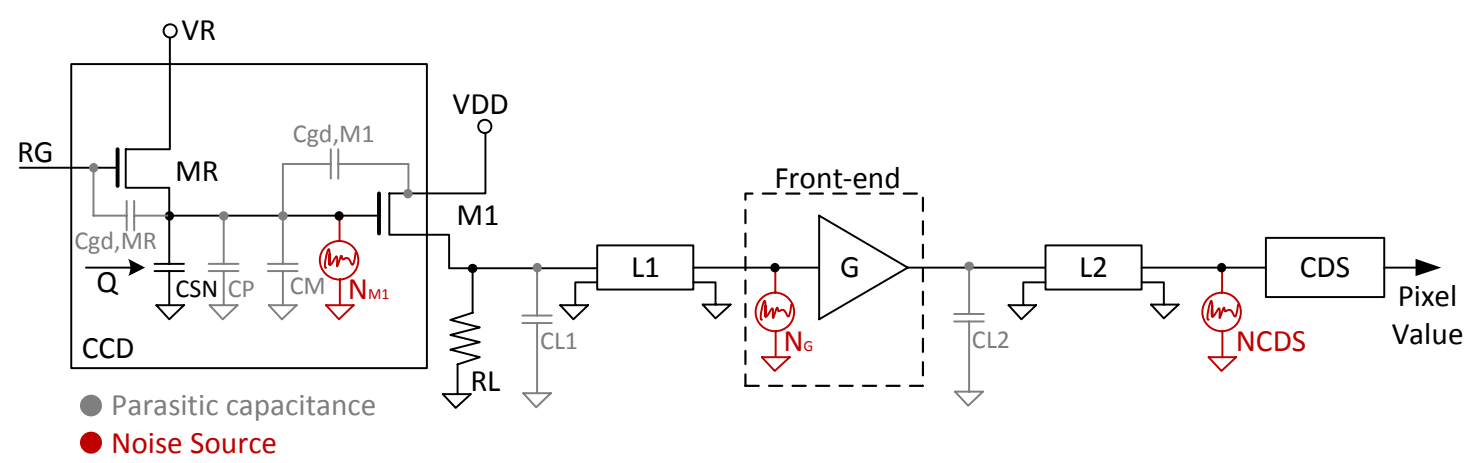

(a)

Figure 4: General CCD read out chain. $C_{P}, C_{M}, C_{g d, M 1}$ and $C_{g d, M R}$ are parasitic capacitances. $C_{P}$ is the capacitance of the physical connection between the $\mathrm{SN}$ and the gate of M1, $C_{g d, M 1}$ and $C_{g d, M R}$ are the gatedrain capacitance of M1 and MR transistor respectively, and $C_{M}$ is the Miller-effect capacitance of $M_{1} . N_{M 1}$, $N_{G}$ and $N_{C D S}$ are voltage sources that add noise to the signal. $N_{M 1}$ is originated in the transistor M1, $N_{G}$ in the preamplifier and $N_{C D S}$ in the circuits of the CDS.

where $C_{T}$ is the total capacitance at the sense node including the parasitic capacitances. M1 is a PMOS transistor in a common drain configuration, hence the source follow the gate with a gain $\approx 1$. The video signal then goes through the line $\mathrm{L} 1$ to the front-end electronics that have a gain $\mathrm{G}$. The line $\mathrm{L} 1$ have a parasitic capacitance $C_{L 1}$ that limits the maximum read-out speed of the CCD [18]. The front-end have a very high input impedance and output impedance low enough to drive a $50 \Omega$ coaxial cable L2 without loosing bandwidth. At the end, the video signal is processed by the correlated double sampling filter (CDS), and digitized. The resulting measurement should be calibrated to get the value of the pixel in electrons [9]. There are two natural ways to improve the SNR, the first one is to increase the signal reducing $C_{T}$ [19], and the second one is to reduce the noise. In this work we have modified the front-end electronics to reduce $N_{G}$.

To keep the CCDs far from potential radioactive material, DAMIC/CONNIE CCDs are bound to a 20" flex cable (L1) [6], and are then connected to the front-end electronics. The current DAMIC/CONNIE front-end electronics was designed for the Dark Energy Survey (DES) experiment. It is a 12 inches flex cable, with a buffer on one end, made of a JFET (J271) source follower in order to reduce the high driver impedance of the CCD video output amplifier and increase the pixel acquisition rate [20]. The other end of the flex is equipped with a pre-amplifier (AD8065) with a gain $\mathrm{G}=2$ which drives the video signal to the Monsoon readout electronics [20].

In CONNIE and DAMIC the images are taken every hour and $\approx 10$ hours respectively, hence a fast readout of the CCDs is not necessary and the readout noise is a priority. Considering this, a modified front end electronics was tested. The JFET source follower was removed, and the preamplifier was modified using a low noise operational amplifier (OPA209) and was polarized in a non-inverting configuration with a gain $\mathrm{G}=4$ to reduce the impact of NCDS an improves the overall SNR.

The results are shown in figure 5. The new front-end improves the noise from $2.46 e^{-}$to $1.70 e^{-}$ for a integration windows of $20 \mu \mathrm{s}$ (20Kpixel/second of readout speed). For integration windows below $15 \mu \mathrm{s}$, the noise of the modified frontend is bigger (dashed curves of figure 5(b)). 


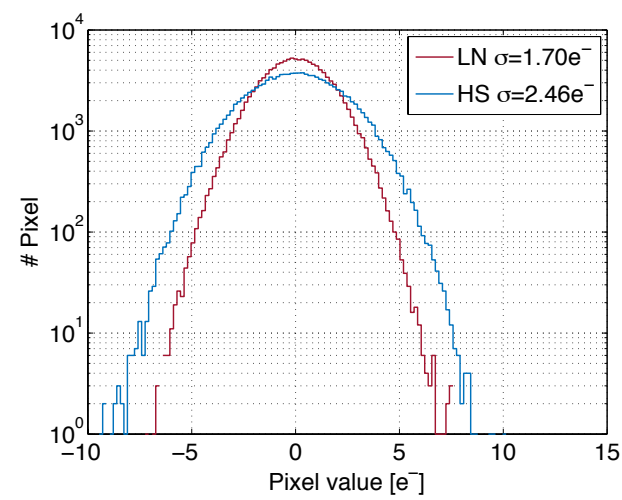

(a)

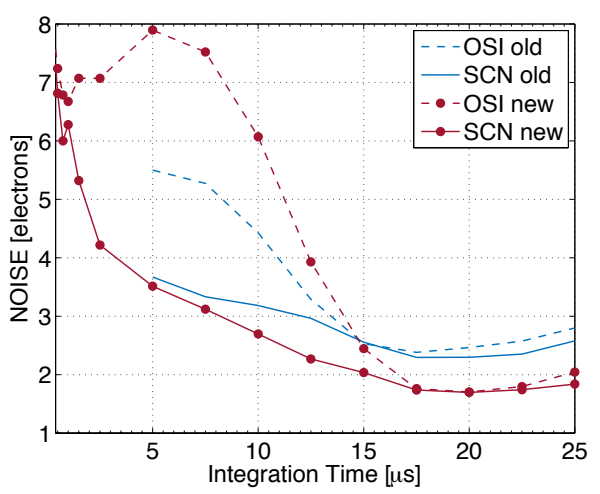

(b)

Figure 5: (a) Overscan pixel distribution for the two front-end electronics: old and new. (b) Noise for different integration times, are the result after applying a overscan subtraction (OSI) and after subtracting the correlated noise $(\mathrm{SCN})$.

While a lower readout noise is achieved with the new front-end, we observed that the system is more sensitive to pickup noise. We attribute this to the length of the cable between the CCD and the front-end electronics, as the new front-end cable is 12" longer. A method to subtract the pickup noise was therefore developed. The pixel array was read out by only one output node $V_{U}$ of the serial register, the video signal of the other output node $\left(V_{L}\right)$ was also recorded. The methods developed in this work is as follow, $V_{L}$ weighted by a parameter $\alpha$ is subtracted to $V_{U}$ to produce $V_{U}^{\prime}, \alpha$ goes from 0 to 1 and is the one that minimize the variance of $V_{U}^{\prime}$. The results of applying the former method for the case of both front-end electronics is shown in figure 5. The subtraction of the correlated noise improve the performance of the new front-end at least over $5 \mu \mathrm{s}$ of integration window.

\section{Conclusion}

In this work a study a point-like events were presented. We observed a dependence of the event size in the image with the amount of the event charge. This is due to the charge repulsion effect and should be taken into account during the estimation of the depth of the event in the bulk. Work is in progress to produce a simulation code taking into account this effect. We have show that CONNIE/DAMIC CCDs are operated without dark-current noise and spurious charge. The electronic readout noise is therefore the main source of noise. A new front-end was developed here. It reduces the readout noise from $2.5 e^{-}$down to $1.7 e-$, allowed to reduce the threshold of the CONNIE/DAMIC experiments to $25 \mathrm{eV}$.

\section{References}

[1] S. E. Holland et al., Fully depleted, back-illuminated charge-coupled devices fabricated on high-resistivity silicon, Electron Devices, IEEE Transactions on 50 (2003) 225-238.

[2] G. Fernandez Moroni et al., Charge coupled devices for detection of coherent neutrino-nucleus scattering, Phys. Rev. D 91 (Apr, 2015) 072001. 
[3] A. Aguilar-Arevalo et al., Results of the engineering run of the coherent neutrino nucleus interaction experiment (connie), Journal of Instrumentation 11 (2016) P07024.

[4] J. Barreto et al., Direct search for low mass dark matter particles with CCDs, Physics Letters B 711 (2012) $264-269$.

[5] A. E. Chavarria et al., $\{$ DAMIC $\}$ at $\{$ SNOLAB $\}$, Physics Procedia 61 (2015) $21-33$.

[6] DAMiC Collaboration collaboration, A. Aguilar-Arevalo et al., Search for low-mass wimps in a $0.6 \mathrm{~kg}$ day exposure of the damic experiment at snolab, Phys. Rev. D 94 (Oct, 2016) 082006.

[7] S. E. Holland et al., Device design for a 12.3-megapixel, fully depleted, back-illuminated, high-voltage compatible charge-coupled device, Electron Devices, IEEE Transactions on 56 (2009) 2612-2622.

[8] S. E. Holland, High-voltage compatible, full-depleted CCD, Sept. 18, 2007.

[9] J. R. Janesick et al., New advancements in charge-coupled device technology: subelectron noise and 4096 x 4096 pixel CCDs, in SC-DL tentative, pp. 223-237, International Society for Optics and Photonics, 1990.

[10] F. Scholze et al., Characterization of a windowless si (li) detector in the photon energy range 0.1-5 kev, Nuclear Instruments and Methods in Physics Research Section A: Accelerators, Spectrometers, Detectors and Associated Equipment 339 (1994) 49-54.

[11] G. F. Moroni et al., Mathematical model of point events in CCD images, in 2015 XVI Workshop on Information Processing and Control (RPIC), pp. 1-6, Oct, 2015. DOI.

[12] E. Gatti et al., Dynamics of electrons in drift detectors, Nuclear Instruments and Methods in Physics Research Section A: Accelerators, Spectrometers, Detectors and Associated Equipment 253 (1987) 393-399.

[13] R. Bellwied et al., Studies of dynamics of electron clouds in star silicon drift detectors, Nuclear Instruments and Methods in Physics Research Section A: Accelerators, Spectrometers, Detectors and Associated Equipment 439 (2000) 507-512.

[14] H. Yousef, Energy dependent charge spread function in a dedicated synchrotron beam pnCCD detector. PhD thesis, Ph. D thesis, Universität Siegen, Siegen, Germany, 2011.

[15] J. Janesick, Scientific Charge-coupled Devices. Press Monographs. Society of Photo Optical, 2001.

[16] W. W. Zhang et al., Optimum signal-to-noise ratio performance of electron multiplying charge coupled devices, World Academy of Science, Engineering and Technology 54 (2009) 264-268.

[17] M. Wei et al., Characterization and optimization of MIT lincoln laboratories CCID20 CCDs, in Astronomical Telescopes \& Instrumentation, pp. 598-607, International Society for Optics and Photonics, 1998.

[18] M. S. Haro et al., Measurement of the read-out noise of fully depleted thick CCDs, in Micro-Nanoelectronics, Technology and Applications (CAMTA), Argentine Conference of, pp. 11-16, IEEE, 2016.

[19] S. Haque et al., Design of low-noise output amplifiers for p-channel charge-coupled devices fabricated on high-resistivity silicon, in IS\&T/SPIE Electronic Imaging, pp. 82980X-82980X, International Society for Optics and Photonics, 2012.

[20] T. Shaw et al., System architecture of the dark energy survey camera readout electronics, in SPIE Astronomical Telescopes + Instrumentation, pp. 77353G-77353G, International Society for Optics and Photonics, 2010. 